\title{
Trasplante cardíaco
}

\section{Heart transplant}

\author{
M. Ubilla, S. Mastrobuoni, A. Martín Arnau, A. Cordero, E. Alegría, J. J. Gavira, M. J. Iriba- \\ rren, T. Rodríguez-Fernández, J. Herreros, G. Rábago
}

\section{RESUMEN}

El trasplante cardíaco es considerado actualmente como el tratamiento de elección en la insuficiencia cardíaca terminal refractaria a tratamiento médico o quirúrgico. Debido a factores como la mayor esperanza de vida de la población y el manejo más eficaz de los síndromes coronarios agudos, cada vez hay un número mayor de personas que padecen fallo cardíaco. Se estima que la prevalencia de la enfermedad en países desarrollados está en torno al 1\%; de éstos, un $10 \%$ está en una etapa avanzada y por tanto son potenciales receptores de un trasplante cardíaco. El problema está en que aún no es posible ofrecer esta modalidad terapéutica a todos los pacientes que la requieren. Por consiguiente, se hace necesario optimizar los resultados del trasplante cardíaco mediante la selección de pacientes, selección y manejo de los donantes, manejo perioperatorio y control de la enfermedad por rechazo del injerto. Desde el primer trasplante efectuado en diciembre de 1967, numerosos avances y cambios se han producido, lo que ha permitido aumentar la supervivencia y calidad de vida de quienes han recibido un nuevo corazón. A continuación se revisarán los aspectos más relevantes del trasplante cardíaco y los desafíos que enfrenta en la actualidad.

Palabras clave. Trasplante cardíaco. Enfermedad crónica del injerto. Rechazo agudo.

\begin{abstract}
A heart transplant is at present considered the treatment of choice in cases of terminal cardiac insufficiency refractory to medical or surgical treatment. Due to factors such as the greater life expectancy of the population and the more efficient management of acute coronary syndromes, there is an increasing number of people who suffer from heart failure. It is estimated that the prevalence of the disease in developed countries is around 1\%; of this figure, some $10 \%$ are in an advanced stage and are thus potential receptors of a heart transplant. The problem is that it is still not possible to offer this therapeutic form to all of the patients that require it. Consequently, it is necessary to optimise the results of the heart transplant through the selection of patients, selection and management of donors, perioperative management and control of the disease due to graft rejection. Since the first transplant carried out in 1967, numerous advances and changes have taken place, which has made it possible to increase survival and quality of life of those who have received a new heart. In this article we review the most relevant aspects of the heart transplant and the challenges that are currently faced.
\end{abstract}

Key words. Heart transplant. Chronic disease of the graft. Acute rejection.

An. Sist. Sanit. Navar. 2006; 29 (Supl. 2): 63-78.

Servicio de Cirugía Cardiovascular y Cardiología. Clínica Universitaria. Pamplona. Navarra.

\section{Correspondencia:}

Gregorio Rábago

Servicios de Cirugía Cardiovascular y Cardiología Clínica Universitaria de Navarra

Universidad de Navarra

E mail: grabago@unav.es 


\section{INTRODUCCIÓN}

Los pacientes que sufren de insuficiencia cardíaca avanzada tradicionalmente han sido sometidos a tratamientos farmacológicos hasta que su condición no puede ser mantenida por estos medios. Un importante avance en esta área ha sido la comprobación de los beneficios aportados por diuréticos, inhibidores de la enzima de conversión y más recientemente, bloqueadores de los receptores beta adrenérgicos. La progresión de la enfermedad a pesar de tales terapias, ha obligado a buscar nuevas alternativas terapéuticas. Así fue como se planteó originalmente la sustitución del corazón enfermo por uno sano. Sin embargo, desde el primer trasplante cardíaco, se han introducido nuevas alternativas para el manejo de la insuficiencia cardíaca avanzada antes de llegar al trasplante. Entre ellas destacan los dispositivos de asistencia ventricular, la resincronización cardíaca, el desfibrilador automático implantable, la cirugía y la terapia celular. Frente a todas estas nuevas opciones es razonable preguntarse cuál es el beneficio de sobrevida que el trasplante puede aportar en la insuficiencia cardíaca avanzada. Para estimarlo, se han comparado los resultados de los pacientes que recibieron un trasplante y de los que fallecieron en lista de espera. En Estados Unidos, según UNOS (United Network for Organ Sharing), la mortalidad de los pacientes en lista de espera alcanzó el 17,2\% en 1999, mientras que la supervivencia de los que fueron trasplantados en igual período fue de $86 \%$; se debe precisar que los pacientes más graves, presentaron una mortalidad del $58,2 \%$ (estado 1, recibiendo inótropos en dosis altas o asistencia ventricular) y 20,5\% (estado 1B, tratados con inótropos en dosis bajas), mientras que los trasplantados en similar condición exhibieron una supervivencia de $84,8 \%$. Algo similar reportó el estudio COCPIT (Comparative outcomes and clinical profiles in transplantation) en 2000 , al encontrar que los pacientes con mayor riesgo de morir en lista de espera según HFSS (Heart Failure Survival Score) eran los que obtenían un mayor beneficio de sobrevida con el trasplante.

No obstante lo anterior, en un análisis retrospectivo de un solo centro, se comparó los resultados del tratamiento de la insuficiencia cardíaca con los del trasplante; lo interesante fue que la cohorte de terapia médica se dividió en dos períodos basándose en los esquemas terapéuticos actuales y antiguos. Se encontró que la sobrevida con tratamiento médico mejoró en el grupo sometido al esquema actual y este grupo, a su vez, tuvo una sobrevida similar a la del grupo trasplantado. Más allá de los sesgos de este estudio, se comienza a plantear que el manejo médico actual de la insuficiencia cardíaca puede obtener resultados similares al trasplante y que se deben revisar los criterios para indicar el trasplante, sobre todo si se considera la dificultad en conseguir donantes.

Pero el tratamiento médico no es el único que ha progresado. En la última década ha aparecido evidencia respecto de la utilidad de la asistencia ventricular mecánica en pacientes con insuficiencia cardíaca terminal. En 2001, el estudio REMATCH demostró que en pacientes rechazados para trasplante, la asistencia ventricular con el dispositivo HeartMate mejoraba la sobrevida al compararlo con un tratamiento médico óptimo (23\% versus $8 \%$ a 2 años, $\mathrm{p}=0,001)$; se debe notar, que los pacientes incluidos en este estudio presentaban un mayor riesgo de mortalidad al compararlos con los destinados a trasplante. Considerando las continuas mejorías en los dispositivos de asistencia ventricular, es posible que en el futuro sus resultados mejoren hasta niveles similares a los del trasplante. Actualmente, hay varios estudios en curso que comparan la asistencia ventricular con el manejo médico.

\section{INDICACIÓN DEL TRASPLANTE CARDÍACO}

El trasplante cardíaco es, en general, el tratamiento de elección para la insuficiencia cardíaca cuando se estima que la supervivencia y calidad de vida no pueden mejorarse con otra alternativa terapéutica tradicional. El problema radica en establecer cuándo las demás opciones de tratamiento no podrán mejorar la sobrevida ni la calidad de la misma. Cuando el tratamiento médico máximo no logra compensar una cardiopatía, el trasplante es una opción. Sin embargo, surge otro problema. 
En las últimas décadas, se ha publicado creciente evidencia sobre la utilidad de nuevas terapias en insuficiencia cardíaca. De ese modo, se ha podido demostrar una mejoría en la sobrevida esperada hasta alcanzar niveles similares a los del trasplante. Por este motivo, se debate el rol del trasplante como alternativa a los tratamientos tradicionales.

Existen indicaciones más específicas dependiendo del contexto de cada enfermedad. La más clara es la disfunción miocárdica severa, irreversible que no responde a tratamiento y cuya expectativa de vida no supera los tres meses (Tabla 1).

En adultos, las indicaciones de trasplante cardíaco se distribuyen de la siguiente manera: cardiopatía isquémica $42 \%$, miocardiopatías $34 \%$, valvulopatías $9 \%$, otras $12 \%$ y retrasplante $3 \%$. En la figura 1 se encuentran reflejados los datos de los grupos de trasplante en su informe del 2005. Las indicaciones de trasplante pediátrico varían un poco con la edad, pero en general las más frecuentes son cardiopatías congénitas $65 \%$, miocardiopatías $29 \%$, retrasplante 1\% y misceláneas 5\%.

Existen numerosos factores que empeoran los resultados del trasplante cardíaco y se consideran contraindicaciones, como hipertensión pulmonar irreversible (mayor a 6 unidades Wood), infección activa y no tratada, infección por $\mathrm{VIH}$, enfermedad irreversible renal, pulmonar o hepática, infarto pulmonar reciente, diabetes mellitus con daño importante de órganos blanco, enfermedad cerebrovascular o vascular periférica, neoplasia maligna en los últimos cinco años, osteoporosis, enfermedad psiquiátrica no compatible con el régimen de vida postrasplante, adicción activa a tabaco, alcohol o drogas.

Tabla 1. Indicaciones para trasplante cardiaco según la American Heart Association y American College of Cardiology.

\section{Indicaciones de trasplante cardíaco. ACC/AHA Practice Guidelines, 2005}

Indicaciones claras

1. Shock cardiogénico refractario al tratamiento médico.

2. Dependencia documentada de soporte inotrópico intravenoso para mantener una adecuada perfusión de órganos.

3. $\mathrm{VO} 2 \mathrm{pico}<10 \mathrm{ml} / \mathrm{kg} / \mathrm{min}$ habiendo alcanzado metabolismo anaeróbico.

4. Síntomas severos de isquemia que limitan de forma consistente la actividad habitual y que no son susceptibles de intervención quirúrgica, revascularización coronaria o intervención coronaria percutánea.

5. Arritmias ventriculares recurrentes refractarias a todas las modalidades terapéuticas.

Indicaciones relativas

1. VO2 pico de $11-14 \mathrm{ml} / \mathrm{kg} / \mathrm{min}$ (o $55 \%$ del estimado) y limitación importante de la actividad diaria del paciente.

2. Isquemia inestable y recurrente no susceptible de otra intervención.

3. Inestabilidad recurrente del balance de fluidos/función renal no debida a un mal cumplimiento del tratamiento médico por parte del paciente.

4. Shock cardiogénico que requiere soporte mecánico (ventilación mecánica, balón aórtico de contrapulsación, asistencia ventricular mecánica) y disfunción orgánica múltiple reversible.

Indicaciones insuficientes

1. Fracción de eyección del ventrículo izquierdo reducida.

2. Historia de síntomas de IC en clase funcional III o IV de la NYHA.

3. $\mathrm{VO} 2 \mathrm{pico}>15 \mathrm{ml} / \mathrm{kg} / \mathrm{min}$ (y mayor del $55 \%$ del estimado) sin otras indicaciones. 


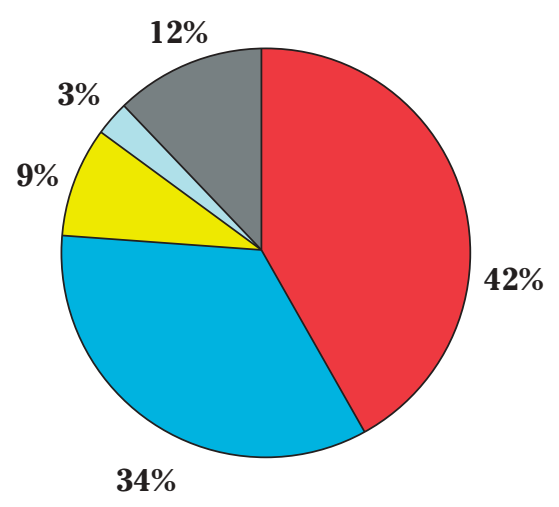

$\square$ CI $\square$ MCDi $\square$ Valv. $\square$ Retx $\square$ Otras

Figura 1. Indicaciones de trasplante cardiaco. Datos del registro nacional de trasplante cardiaco.

\section{SELECCIÓN Y MANEJO DEL DONANTE}

Inicialmente los criterios de selección del donante eran muy restrictivos. Con el paso del tiempo y ante la falta de un número suficiente de donantes, estos criterios se han expandido. Hay experiencias exitosas de trasplante con donantes mayores de 55 años en casos seleccionados. El tamaño del corazón donado debe ser adecuado para el receptor; en general, cuando el donante tiene un peso y talla promedio, lo más probable es que funcione bien en la mayoría de los receptores. Hay condiciones bajo las cuales no es conveniente aceptar un donante. Ejemplo de esto es la cardiopatía estructural, isquémica y las arritmias ventriculares refractarias; la presencia de neoplasia maligna (no del sistema nervioso central) o infección no controlada contraindican el uso del corazón como injerto.

Una vez aceptado un donante, el manejo previo al trasplante es fundamental para optimizar los resultados. La muerte cerebral desencadena un conjunto de procesos que, en conjunto con la lesión que inicialmente llevó a la muerte, ponen en riesgo la viabilidad del corazón. Entre estos, destaca la activación adrenérgica que potencial- mente puede generar isquemia subendocárdica. Los mediadores inflamatorios producen depresión miocárdica. A esto se suma una tendencia a la hipovolemia. Para controlar esto, es necesario hacer una estricta monitorización de las variables hemodinámicas, respiratorias y metabólicas. Se ha demostrado que la administración de sustitución hormonal con corticoesteroides, insulina, hormona tiroidea y vasopresina mejora los resultados.

\section{Características deseables en el donante}

1. Edad inferior a 45 años en el hombre y a 50 en la mujer.

2. Tamaño idóneo. El peso del donante o su superficie corporal no debe ser inferior al $25 \%$ de la del receptor.

3. Antecedentes y examen cardiológico normales. En mayores de 40 años, el ecocardiograma debe realizarse siempre que sea posible.

4. Compatibilidad del grupo sanguíneo ABO.

5. Estabilidad hemodinámica tras la normalización de la volemia y con bajas dosis de fármacos inotrópicos.

6. Ausencia de enfermedades transmisibles. Serología negativa para hepatitis y SIDA, teniendo en cuenta que el resultado puede ser falso negativo por ventana inmunológica o debido a la hemodilución, razón por la que se debe conservar la muestra de serología durante un largo periodo de tiempo.

7. Ausencia de infección activa o neoplasia con posibilidad de diseminación. Se recomienda la extracción de cultivos de sangre, de orina y de exudado traqueal; la determinación de HCG y en casos sospechosos de CEA, -fetoproteína y antígeno prostático específico.

8. Sin antecedentes cardiovasculares, ni coronarios, hipertensión de larga evolución, sin antecedentes de parada cardíaca. No debe tener historia de diabetes insulinodependiente. Sin adicción al tabaco. Sin dislipemias conocidas.

9. Tiempo previsto de isquemia del injerto inferior a 4-5 horas. 
10. Prueba cruzada. Se realiza retrospectivamente en todos los casos y prospectivamente:

- Si el receptor tiene positividad de anticuerpos antilinfocitarios (panel $>20 \%$ ).

- Si el paciente es transfundido después de tener un panel negativo.

- Si retrospectivamente existieron anticuerpos y posteriormente se negativizaron.

\section{TÉCNICA QUIRÚRGICA}

En los años 60, Lower y Shamway describieron la técnica clásica del trasplante cardíaco. Consiste en la realización de cuatro anastomosis: aurículas derechas e izquierda, arteria pulmonar y aorta. Durante 30 años se ha considerado la técnica estándar, aunque era común observar la aparición de insuficiencia de las válvulas aurículoventriculares atribuida a la distorsión de la anatomía auricular. Por ello, en los 90 se introdujeron técnicas alternativas. Yacoub y Sievers describieron la técnica bicava, en que se conserva la aurícula derecha y se efectúa anastomosis de cada vena cava por separado. Posteriormente se reportó la técnica de trasplante total, cuya característica es la preservación de la aurícula izquierda con anastomosis de las venas pulmonares del donante y receptor.

En varios estudios se ha comparado los resultados de las técnicas clásicas y bicava.

En un estudio prospectivo y randomizado de 201 casos, con técnica bicava a 1,3 y 5 años se demostró una supervivencia de $87 \%, 82 \%$ y $81 \%$, mientras que con técnica clásica se obtuvieron supervivencias de $74 \%, 70 \%$ y $62 \%$ respectivamente ${ }^{1}$.

Las dimensiones auriculares han sido evaluadas en varios estudios, encontrándose siempre menores tamaños con técnica bicava $^{2-5}$. También se han encontrado mejores resultados con la técnica bicava en cuanto a la competencia de las válvulas aurículoventriculare ${ }^{6-9}$, menor incidencia de arritmias y reducción de la necesidad de marcapaso $^{10-12}$ lo que se traduce en una estancia hospitalaria más corta ${ }^{3,4}$. Además, en el periodo perioperatorio, los pacientes sometidos a la técnica bicava requieren menos vaospresores y tienen menor pérdida de sangre ${ }^{13}$ que los operados con técnica clásica. Se ha planteado que la técnica bicava puede producir estenosis de las anastomosis en las venas cavas, sin embargo en la práctica esto no ha sido un problema ${ }^{11}$.

Hay menos trabajos que comparen las técnicas clásica y total, debido a que esta última se realiza en menos centros (8\% de los trasplantes según ISHLT).

Con técnica total, la supervivencia reportada ha sido mejor que con la clásica, con menor incidencia de regurgitación de las válvulas aurículoventriculares ${ }^{14}$, menos arritmias, menos requerimientos de marcapasos, menos trombos auriculares y eventos embólicos ${ }^{15-17}$.

\section{MANEJO DESPUÉS DEL TRASPLANTE}

\section{Inmunosupresión}

La inmunosupresión ha determinado una mejoría sostenida en el pronóstico de los pacientes sometidos a trasplante de órganos sólidos. La introducción de fármacos eficaces como ciclosporina, azatioprina y corticoides, ha permitido el desarrollo de los programas de trasplante cardíaco. Durante los últimos 15 años han aparecido nuevos fármacos que han ampliado las opciones para inmunosuprimir en trasplante; el problema de hoy es encontrar la combinación de fármacos más adecuada.

Los fármacos inmunosupresores disponibles hoy se pueden clasificar en:

- Inductores: OKT3, timoglobulinas y antagonistas de los receptores de IL-2 (daclizumab y basiliximab).

- Anticalcineurínicos: ciclosporina y tacrolimus.

- Antimetabolitos o inhibidores de la síntesis de purinas: micofenolato mofetil y azatioprina.

- Corticoesteroides.

- Antiproliferativos o inhibidores de mTOR: sirolimus y everolimus.

Estos fármacos se pueden combinar de diversas formas, constituyendo las pautas de inmunosupresión, que se pueden clasificar según su indicación en: inducción, mantención y rechazo. 
El régimen de inducción tiene por finalidad bloquear la respuesta inmune en el período inicial del trasplante (cuando es más intensa), pero con el costo de una mayor incidencia de infecciones y neoplasias. Tradicionalmente se han empleado agentes linfolíticos como timoglobulina por 7 a 14 días. En los últimos diez años se han introducido los anticuerpos bloqueadores de los receptores de IL-2, que parecen reducir los episodios de rechazo sin aumentar mucho las infecciones ${ }^{18,19}$. En algunos centros la inducción se reserva para los pacientes con insuficiencia renal crónica (porque permite diferir el uso de anticalcineurínicos) y alto riesgo de rechazo. Según el registro de ISHLT, aproximadamente el 50\% de los receptores de trasplante cardíaco ha recibido algún inductor. Para el régimen de mantención se suele emplear un anticalcineurínico, un antimetabolito y un esteroide; en el último tiempo han surgido nuevas pautas para reducir la nefrotoxicidad de los anticalcineurínicos y los problemas metabólicos de los corticoides, agregando un inhibidor de mTOR para reducir las dosis o eliminar alguno de los otros dos. Existe un creciente uso de pautas en que se elimina el corticoide entre 6 meses y 2 años sin que ello implique un mayor riesgo de rechazo y con reducción de los efectos adversos ${ }^{20}$. En la figura 2 se aprecia los diferentes regímenes inmunosupresores seguidos en la actualidad por nuestros pacientes.
El tratamiento de los episodios de rechazo depende de la magnitud del episodio (si hay o no compromiso hemodinámico) y del tipo (celular o humoral).

Los efectos generales de los inmunosupresores se pueden clasificar en 3 categorías:

- Efectos inmunes deseados (inmunosupresión).

- Efectos adversos inmunes: infecciones y neoplasias.

- Efectos adversos no inmunes: diabetes, HTA, nefrotoxicidad, hiperlipidemia, osteoporosis, neutropenia, anemia, trombocitopenia, hiperuricemia, diarrea, alteración en la cicatrización, etc.

El uso de fármacos inmunosupresores supone un riguroso control de los niveles plasmáticos de algunos de ellos (ciclosporina, tacrolimus, micofenolato mofetil, sirolimus, everolimus) y una constante vigilancia de las otras terapias farmacológicas ya que existen frecuentes interacciones que modifican los efectos.

\section{Prevención y manejo de infecciones}

Las infecciones son responsables de una parte importante de la morbimortalidad entre los pacientes trasplantados ${ }^{21}$. Globalmente, la incidencia de infecciones oscila entre el $30-60 \%$ y la mortalidad relacionada es del 4-15\%. Según el tipo de agente etioló-

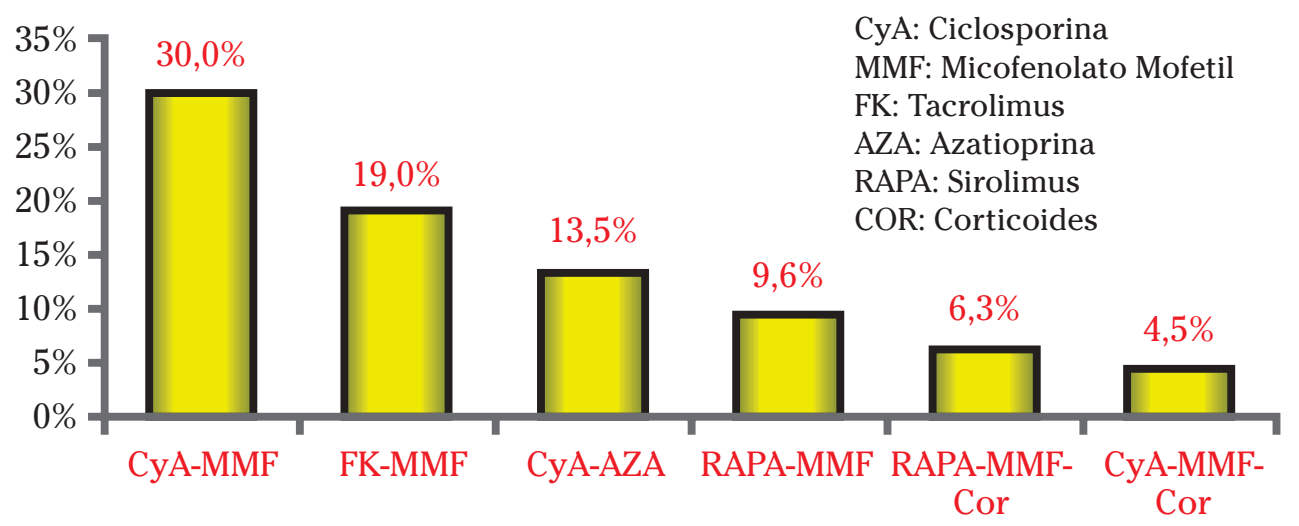

Figura 2. Tratamiento actual de los pacientes trasplantados cardiaco en la Clínica Universitaria. 
gico, su frecuencia es: bacterianas 43-60\%, virales $40-45 \%$, hongos y protozoos $8-14 \%$. Las infecciones más importantes suelen aparecer durante los 3 primeros meses y su localización más común es en los pulmones y el sitio quirúrgico.

Los agentes etiológicos específicos dependen del momento en que aparezca la infección.

- Primer mes: bacterias nosocomiales en herida operatoria, pulmón, vías urinarias y catéteres; su pronóstico es grave.

- Segundo al sexto mes: bacterias oportunistas, citomegalovirus (CMV), hongos y protozoos; en esta etapa la inmunidad celular está más reducida.

- Después del sexto mes: infecciones adquiridas en la comunidad; micobacterias, criptococo, micosis endémicas (primoinfecciones o reactivaciones).

El 90\% de las infecciones bacterianas se presentan durante el primer mes. Los agentes más frecuentes son estafilococo y bacilos Gram negativos (E. coli, Serratia marcescens, Pseudomonas y Acinetobacter). Los factores que condicionan un mayor riesgo de adquirir una infección bacteriana son edad avanzada, diabetes, desnutrición, EPOC y fallo renal. La prevención de infecciones precoces es un punto fundamental dado el impacto que tienen en la mortalidad. Ésta debe comprender una profilaxis antimicrobiana apropiada que, entre otros aspectos, ha de considerar la colonización con flora nosocomial, uso de ventilación mecánica previa al trasplante, empleo de dispositivos de asistencia ventricular, catéteres, drenajes, tratamientos antimicrobianos y resultados de cultivos recientes. Más tardíamente pueden aparecer infecciones por bacterias intracelulares como Legionella, Listeria, Nocardia, Pneumocystis carinii y micobacterias. Después del sexto mes, aparecen las bacterias de la comunidad, salvo que al paciente se le aumente la inmunosupresión por rechazo.

Las infecciones víricas ocupan el segundo lugar en cuanto a frecuencia. Se han identificado como factores de riesgo para su aparición: inmunosupresión, donante con serología positiva para CMV, virus Epstein-Barr (EBV) u otros. Para detectar precozmente los pacientes que están en riesgo de adquirir una infección vírica, se efectúan estudios serológicos de rutina, que en los donantes incluyen virus de la inmunodeficiencia humana (VIH), virus de la hepatitis B (VHB), virus de la hepatitis C (VHC), HTLV I y II, CMV y VEB: en los receptores se hacen determinaciones para VIH, VHA, VHB, VHC, VVZ, CMV y EBV. También para prevenir, en los candidatos a trasplante se realizan vacunaciones para cubrir los virus que pueden provocar infecciones con más frecuencia: VHB, VHA, gripe, varicela, sarampión, parotiditis, rubéola y polio. Después del trasplante se puede repetir las vacunas contra la gripe, hepatitis A y B y polio (VPI).

Los agentes que con mayor frecuencia se aíslan en pacientes trasplantados cardíacos son virus herpes simple (VHS), CMV y virus varicela-zóster (VVZ). Otros menos habituales son herpes humano tipo 6, VHB, VHC, Influenza A y B, Parainfluenza, Virus respiratorio sincitial (VRS) y Parvovirus $B 19$. Todos ellos dan origen a variados cuadros clínicos que, en general, afectan a piel, mucosas, tracto respiratorio, hígado y tracto gastrointestinal.

Es distintivo de algunos virus el momento en que provocan infección después del trasplante. En el primer mes suele aparecer VHS. Entre el segundo y tercer mes se presentan las infecciones por CMV; después de los 6 meses se ven infecciones por VVZ. Citomegalovirus sigue siendo una de las infecciones más importantes en la población trasplantada, aunque su mortalidad es baja (menos del 1\%). Sin embargo, su presencia se asocia a un mal pronóstico a largo plazo y mayor frecuencia de enfermedad vascular del injerto.

Las infecciones fúngicas son menos frecuentes (2-15\% micosis invasivas), pero característicamente tienen una alta mortalidad (30-100\%); Se han descrito dos tipos de infecciones fúngicas ${ }^{22}$.

Micosis invasoras oportunistas, causadas por Candida, Aspergilus, Criptococus neoformans y mucorales. Por regla general se adquieren en el ambiente hospitalario. Candida se manifiesta como una micosis diseminada. Aspergilus es responsable de 
más del 60\% de las infecciones fúngicas en esta población de pacientes y su localización más común es el pulmón.

Las micosis pulmonares o diseminadas representadas por histoplasmosis, coccidioidomicosis y blastomicosis son adquiridas en la comunidad.

El pronóstico de las infecciones fúngicas, de por sí sombrío, depende de la velocidad con que se efectúe el diagnóstico y se instaure el tratamiento; para ello, es necesario tener un alto índice de sospecha clínica y realizar las pruebas necesarias: cultivos, serología y biopsias, lo que dependerá del agente que se pretenda identificar. El tratamiento es difícil, porque el mejor antifúngico, anfotericina, es nefrotóxico e interactúa con ciclosporina, lo que puede magnificar el daño renal.

Se recomienda la prevención de la candidiasis oral mediante el uso de enjuagues orales de nistatina por 6 a 12 meses; lo mismo se ha visto que resulta útil en los candidatos a trasplante que están sometidos a un dispositivo de asistencia ventricular $^{23}$. También se ha recomendado el uso de profilaxis en pacientes que presentan un alto riesgo de micosis sistémica, con fluconazol, itraconazol o anfotericina. Sin embargo, una revisión sistemática reciente, no encontró evidencia suficiente pera apoyar el uso profiláctico de antifúngicos en recipientes de trasplante cardíaco ${ }^{24}$.

\section{Vigilancia y seguimiento}

Los pacientes que han superado el período postoperatorio precoz, normalmente son dados de alta e ingresan a un programa de seguimiento ambulatorio. Sus objetivos son detectar precozmente el rechazo, valorar la función del injerto, hacer una evaluación general del estado de salud del receptor y descartar posibles complicaciones relacionadas con el trasplante. El protocolo de vigilancia puede variar dependiendo del centro, pero en términos generales implica la realización de revisiones cuya periodicidad se va reduciendo en la medida que pasa el tiempo hasta llegar a una frecuencia de dos veces al año. Dentro de estas revisiones, además de la valoración clínica básica, se incluye la biopsia endomiocárdica y una serie de pruebas como análisis de sangre, electrocardiograma, ecocardiograma, radiografía de tórax y cateterismo cardíaco.

La biopsia endomiocárdica es considerada el método de elección para el diagnóstico del rechazo.

\section{Rechazo}

El rechazo del injerto es una de las complicaciones más temidas en el trasplante. Su incidencia y gravedad se ha reducido conforme han aparecido mejores regímenes de inmunosupresión. No obstante, los episodios de rechazo grave siguen constituyendo una seria amenaza para la vida de lo receptores de trasplante cardíaco.

Se han reconocido tres tipos de rechazo: hiperagudo, agudo celular y humoral y crónico.

\section{Rechazo hiperagudo}

Es causado por anticuerpos preformados por el receptor contra antígenos $\mathrm{ABO}$, HLA o del endotelio del donante. Su inicio es violento, dentro de minutos u horas desde que se restablece la circulación coronaria. Entre los factores de riesgo identificados cabe destacar la politransfusión (multíparas y politransfundidos) y el trasplante con incompatibilidad ABO. Su evolución es rápida, con un brusco deterioro de la función del injerto que, casi invariablemente, lleva a la muerte; afortunadamente en la actualidad es raro, debido a las precauciones que se toman en el período perioperatorio.

\section{Rechazo agudo celular}

Ocurre habitualmente dentro de los primeros 3 a 6 meses de realizado el trasplante; cuando ocurre después de los primeros 6 meses, suele verse en quienes tuvieron episodios previos de rechazo moderado o severo, reciente reducción en la inmunosupresión, infección intercurrente o no han cumplido con la pauta de inmunosupresores. Como factores de riesgo se ha identificado el tiempo después del trasplante (alcanza un máximo al mes y luego declina), tipo de inmunosupresión (especialmente OKT3) y otros 
(recipiente joven, donante o recipiente mujer, recipiente de raza negra, número de incompatibilidades de HLA donantereceptor $)^{25-27}$. Su frecuencia ha disminuido desde $70-85 \%$ a $40 \%$ entre 1982 y 2004 según ISHLT cifras que pueden estar infravaloradas, ya que este registro no toma en consideración los rechazos leves a moderados. El rechazo agudo provoca el $7 \%$ de las muertes en el primer mes, $12 \%$ entre 31 días y 1 año y $10 \%$ entre 1 y 3 años La mayor parte de los casos no es grave; sólo el $5 \%$ de los casos se presenta con compromiso hemodinámico ${ }^{28}$. Pueden haber signos de fallo izquierdo (disnea), derecho (dolor abdominal, edema) o arritmias auriculares ${ }^{29}$. El diagnóstico se efectúa mediante biopsia endomiocárdica que se hace si la clínica sugiere un episodio de rechazo o como parte del protocolo de vigilancia de cada centro. La clasificación y tipo viene especificada en la tabla 2 de acuerdo a la clasificación de la ISHLT $^{30}$. Se debe tener precaución al interpretar las biopsias, ya que hay lesiones que histológicamente se parecen al rechazo pero no lo son: lesiones Quilty, infección por CMV y toxoplasma, lesión por isquemia-reperfusión e infiltración miocárdica por trastornos linfoproliferativos. Para obviar la necesidad de realizar biopsias seriadas, se han hecho varios intentos por identificar un marcador de rechazo no invasivo, pero con malos resultados: troponina $\mathrm{T}$, receptores solubles de IL2, cambios en el electrocardiograma, ecocardiograma, imáge- nes con linfocitos marcados, anexina-V o antimiosina y expresión génica de linfocitos en sangre periférica. El tratamiento de un episodio de rechazo agudo celular depende de dos cosas: magnitud histológica y compromiso clínico-hemodinámico. Según eso, se modulará la intensidad de las alternativas terapéuticas: esteroides (orales o intravenosos), agentes antilinfocíticos policlonales (ATGAM o timoglobulina) o monoclonales (OKT3, daclizumab y basiliximab) y cambios en los inmunosupresores orales. Es necesario tratar aquellos con compromiso hemodinámico o los grados $3 \mathrm{~A}, 3 \mathrm{~B}$ y 4 . El grado 2 depende de los centros y en la mayoria de los casos se ajusta el tratamiento. Por regla general los grados $1 \mathrm{~A} \mathrm{y}$ 1B no suelen tratarse (Tabla 2).

\section{Rechazo agudo humoral (vascular)}

Puede ocurrir días o semanas después del trasplante ${ }^{31,32}$. Uno de sus sellos es que pueden ocurrir episodios de rechazo con compromiso hemodinámico y sin evidencias de infiltración celular en la biopsia. También se ha visto que los pacientes que presentan rechazo con compromiso hemodinámico y un bajo score ISHLT, tienen peores resultados que aquellos con un alto score ${ }^{33}$. Estos episodios de rechazo con compromiso hemodinámico y biopsia de bajo grado, pueden ser explicados por un error de muestreo (falso negativo) y por rechazo humoral; por el contrario, pueden encontrarse biopsias de alto grado sin evidencias de rechazo

Tabla 2. Clasificación del rechazo celular. En la columna de la izquierda esta la nueva clasificación de la ISHLT.

\begin{tabular}{ccl}
\hline \multicolumn{2}{l}{ GRADO ISHLT } & CARACTERÍSTICAS \\
\hline 0 & 0 & Ausencia de rechazo \\
\hline \multirow{3}{*}{1} & 1A & $\begin{array}{l}\text { Rechazo celular agudo. Infiltrado perivascular o intersticial } \\
\text { focal de grandes linfocitos sin necrosis }\end{array}$ \\
& 1B & Rechazo agudo leve con infiltrado linfocitario difuso sin necrosis. \\
& 2 & Rechazo celular agudo con infiltrado linfocitaro focal. Puede existir daño miocítico. \\
\multirow{2}{*}{2} & 3A & Infiltrado agresivo multifocal con o sin daño miocítico. \\
& 3B & Proceso inflamatorio difuso severo con necrosis miocítica. \\
\hline \multirow{2}{*}{3} & 4 & Infiltrado inflamatorio polimorfo difuso, con edema, hemorragia, vasculitis. \\
& &
\end{tabular}


clínico, lo que ha generado controversia sobre la necesidad de aumentar la inmunosupresión ${ }^{34}$. El rechazo humoral está mediado por anticuerpos más que por células, dirigidos contra antígenos HLA o endoteliales del donante. Se produce con mayor frecuencia en mujeres, pacientes con un panel elevado de anticuerpos reactivos en el screening, cross-match positivo, recipientes seropositivos para CMV y sensibilizados para OKT3 ${ }^{32}$. Afecta al $7 \%$ de los pacientes trasplantados, se asocia con severa disfunción ventricular izquierda (47\% de los casos) $)^{35}$ y con mayor frecuencia de rechazo crónico. Para realizar el diagnóstico, la ISHLT ha propuesto pautas, demostrando inmunoglobulinas y complemento en las paredes de los vasos o células endoteliales hinchadas en la biopsia endomiocárdica. El tratamiento es algo más controvertido debido a la ausencia de evidencia sólida; se recomienda la intensificación o modulación de la inmunosupresión mediante inmunoglobulina intravenosa, ciclofosfamida y plasmaféresis $^{36}$.

\section{Rechazo crónico}

Se le conoce como enfermedad vascular del injerto (EVI). Según ISHLT, entre 1994 y 2004 la incidencia de EVI fue 9\% a 1 año, 34\% a 5 años y $48 \%$ a 8 años; estas frecuencias fueron mayores en los pacientes con enfermedad coronaria. La incidencia de EVI precoz (dentro de los primeros 3 años) fue mayor en los trasplantados entre 1996 y 1998 que en el período 2001-2002 ${ }^{37}$. En el registro de la Cardiac Transplant Research Database, se evaluaron 2.609 pacientes trasplantados entre 1990 y 1995; a los 5 años se encontró EVI en $42 \%$ y hubo un $7 \%$ de muertes o retrasplantes por cardiopatía isquémi$\mathrm{ca}^{38}$. Según ISHLT, la EVI es responsable del $13 \%$ de las muertes después del primer año de trasplante, constituyéndose en la primera causa de muerte en ese período.

Un problema importante con la EVI es que clínicamente es difícil de detectar, dado que el corazón trasplantado, al estar denervado, no manifiesta la isquemia igual que un corazón nativo. Por este motivo, se ha intentado establecer un sis- tema de vigilancia y diagnóstico precoz basado en pruebas eficientes. El diagnóstico se establece mediante coronariografía y especialmente con la ayuda de ultrasonido intravascular (IVUS); este método estima la EVI mediante la medición del máximo grosor intimal (maximal intimal thickness- MIT-) y del índice intimal (medido a partir de las áreas luminal e intimal). Con este método, se ha identificado hasta un $80 \%$ de engrosamiento intimal al primer año de trasplante ${ }^{39}$. El IVUS tiene un rol predictor de los resultados, avalado por estudios que demuestran que los pacientes que presentan un MIT mayor a 0,5 mm tienen más mortalidad, pérdida del injerto, IAM y eventos cardíacos mayores no fatales; asimismo, los que tienen un aumento del MIT sobre 0,5 mm en 1 año (entre 20 y $37 \%$ ), también presentan similares problemas ${ }^{40,41}$.

Las lesiones de la EVI son diferentes a las de la ateroesclerosis común de las arterias coronarias, dado que corresponden a un engrosamiento intimal de distribución difusa, concéntrico, sin calcificación de las placas, que predomina en la porción media y distal de los vasos; en cambio la ateroesclerosis coronaria suele afectar la porción proximal de los vasos, es excéntrica y focal $^{42}$.

La patogenia de la EVI involucra factores inmunes y no inmunes. Entre los factores inmunes descritos están la discordancia HLA, la activación de linfocitos T, activación del endotelio, citoquinas, anticuerpos y rechazo humoral. Entre los factores no inmunes están hiperlipidemia, CMV, diabetes, cardiopatía isquémica, fibrinolisis, disfunción endotelial, sistema renina-angiotensina, deficiencia de óxido nítrico sintetasa y endotelinas.

Se han establecido factores de riesgo inmunes (número y severidad de episodios de rechazo) y no inmunes (infección por CMV, edad/sexo, diagnóstico pretrasplante, lesión por isquemia-reperfusión); también se ha visto que los clásicos factores de riesgo cardiovascular aumentan el riesgo de EVI: tabaco, obesidad, diabetes, HTA e hiperlipidemia ${ }^{43}$.

Actualmente existen dos estrategias para controlar la EVI: prevención y trata- 
miento de los casos establecidos. Dentro de las medidas preventivas está el manejo de la hiperlipidemia con estatinas, que han demostrado mejorar la supervivencia, reducir la incidencia de EVI y reducir los niveles de colesterol ${ }^{44,45}$ no está claro que el uso de fibratos sea tan beneficioso como las estatinas y además, existe preocupación sobre su uso combinado, dado que ambos tienen el potencial de producir rabdomiolisis. Diltiazem resultó beneficioso en evitar la aparición de EVI en un estudio randomizado $^{46,47}$. Un estudio ha mostrado beneficio de la suplementación con vitaminas antioxidantes ( $\mathrm{C}$ y E) en evitar la aparición de EVI detectada por IVUS ${ }^{48}$. Los nuevos agentes antiproliferativos sirolimus y everolimus, han demostrado utilidad en la prevención de EVI al compararlos con inmunosupresión basada en azatioprina $^{49,50}$.

El tratamiento de la EVI contempla las siguientes medidas:

- Cambios en la inmunosupresión: se ha visto que intensificar el régimen de inmunosupresión puede evitar que progrese la EVI e incluso inducir su regresión $^{51}$. Sirolimus también ha mostrado ventajas en controlar la EVI ${ }^{52}$.

- Se ha visto que en lesiones susceptibles de tratamiento percutáneo, la angioplastia y especialmente el uso de stents permiten mantener permeables arterias con EVI; sin embargo, no se conoce su impacto sobre la viabilidad del injerto ni sobre la supervivencia. Tampoco se sabe cuál es el rol de los stents liberadores de drogas ${ }^{53}$.

- La cirugía de revascularización coronaria se considera una opción solo en casos seleccionados, ya que la naturaleza difusa de la EVI hace poco probable que este tipo de revascularización sea útil ${ }^{54}$.

- El retrasplante es la única medida para solucionar definitivamente una EVI; sin embargo, el pronóstico de los pacientes retrasplantados es peor que el de los trasplantados "de novo" (48 versus $79 \%$ de supervivencia a 1 año) y tienen una frecuencia de 13\% de EVI a 3 años (similar a los trasplantes de novo).
- La terapia génica es una alternativa promisoria pero aún no explorada clínicamente; en estudios animales la introducción de genes de tPA y óxido nítrico sintetasa ha permitido reducir la aparición de $\mathrm{EVI}^{55,56}$.

\section{NEOPLASIAS MALIGNAS}

Son una de las principales causas de muerte entre los trasplantados y una consecuencia del uso de inmunosupresores. Los trasplantados tienen un riesgo 10 a 100 veces mayor de desarrollar cáncer que la población general (5-6\% incidencia general).

Los tumores más frecuentes son los de piel y los linfomas; de estos últimos, especialmente los asociados a infección por EBV. En trasplante cardíaco los linfomas son más frecuentes que en trasplante renal, lo que puede deberse a una inmunosupresión más intensa. Se espera que en la medida que aumente la expectativa de vida de los trasplantados, aumente también la incidencia de tumores y de aquellos que son prevalentes en la población general.

Se ha planteado que la causa de una mayor incidencia de neoplasias sería la inmunosupresión por dos mecanismos: disminución de la inmunovigilancia y efecto oncogénico directo.

Es difícil saber cuál es el impacto de cada droga inmunosupresora en la aparición de cáncer, debido a que cada paciente recibe varias drogas. Ciclosporina y tacrolimus tienen similares efectos sobre la aparición de neoplasias ${ }^{57}$. Hay algunos datos que apuntan a que micofenolato mofetil sería menos oncogénico que azatioprina; pero no hay evidencia sólida para establecer una diferencia ${ }^{58}$. En algunos estudios se ha visto que sirolimus tiene un efecto protector frente a los tumores e incluso, se ha descrito regresión de algunas neoplasias al cambiar el régimen de inmunosupresión por uno que lo contenga; asimismo, se ha descrito que sirolimus induce la regresión del sarcoma de Kaposi en pacientes con trasplante renal ${ }^{59-61}$.

Estas neoplasias responden a cambios o reducción en la inmunosupresión; los linfomas que expresan CD20, exhiben una buena respuesta a rituximab. 


\section{RESULTADOS}

Desde julio de 1984 hasta junio de 2006 se han realizado en nuestro centro un total de 228 trasplantes cardíacos. La edad media de dicha población en el momento del trasplante era de $51,4 \pm 15$ años siendo la mayoría varones (91,5\%). El trasplante se realizó de forma electiva en el 83\% de los casos siendo urgente en el $27 \%$. La causa principal para el trasplante fue la cardiopatia isquémica en el $49 \%$ de los casos seguido de la cardiomiopatía dilatada (31,6\%), cardiopatía valvular $(6,6 \%)$ y retrasplante $(4,71 \%)$. La mortalidad a 30 días en trasplante cardíaco es de un 5 a un $10 \%$, estando el $82,5 \%$ de los enfermos vivos en un año y un $75,3 \%$ a los 5 años, un 58,9 a los 10 años y un $44,1 \%$ a los 15 años del trasplante (Fig. 3). En la actualidad, la supervivencia media del corazón supera los 10 años. El fracaso primario del injerto es la complicación más temprana; aunque a veces sea reversible, puede requerir asistencia circulatoria o incluso retrasplante. El rechazo y las infecciones son responsables por la mayor parte de las muertes en los primeros 6 meses. La patología tumoral y el rechazo crónico representan la complicación más tardía. En la figura 4 están representadas las causas más frecuentes de mortalidad tras el trasplante durante toda su evolución.

\section{CONCLUSIONES}

En la actualidad el trasplante cardíaco ha demostrado su eficacia tras más de 30 años de experiencia. Sin duda, es el procedimiento terapéutico en la insuficiencia cardiaca terminal que más favorablemente afecta a la supervivencia de estos pacientes. La escasez de donantes sigue limitando el impacto de esta terapéutica. Hay que continuar informando y sensibilizando a la población sobre la importancia de la donación. En el trasplante de órganos sólidos y particularmente en el trasplante cardiaco convergen una cantidad importante de profesionales de diversas especialidades que aportando su experiencia, consiguen que este tratamiento, sea considerado como uno de los más especializados y sofisticados. Aunque mucho se ha conseguido, es necesario continuar investigando y desarrollando nuevas drogas y estrategias para poder ofertar a más pacientes con insufi-

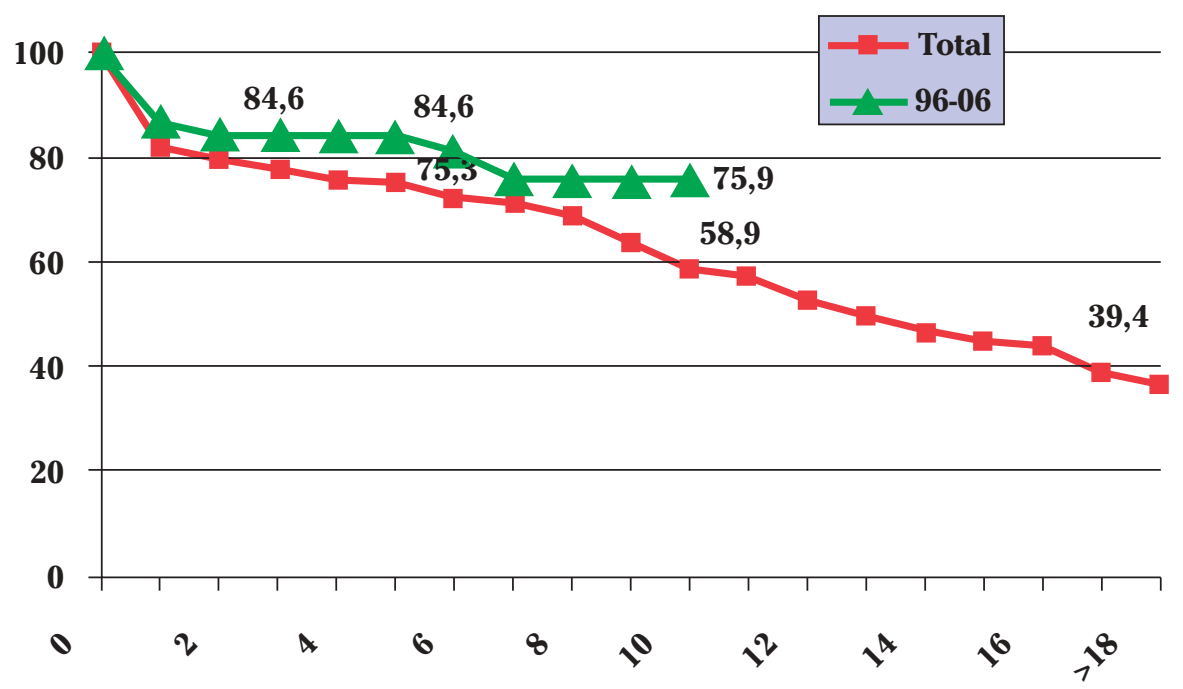

Figura 3. Curva de supervivencia actuarial de los pacientes trasplantados cardiacos desde 1984 hasta 2006. La curva superior representa la experiencia de los 10 últimos años. 


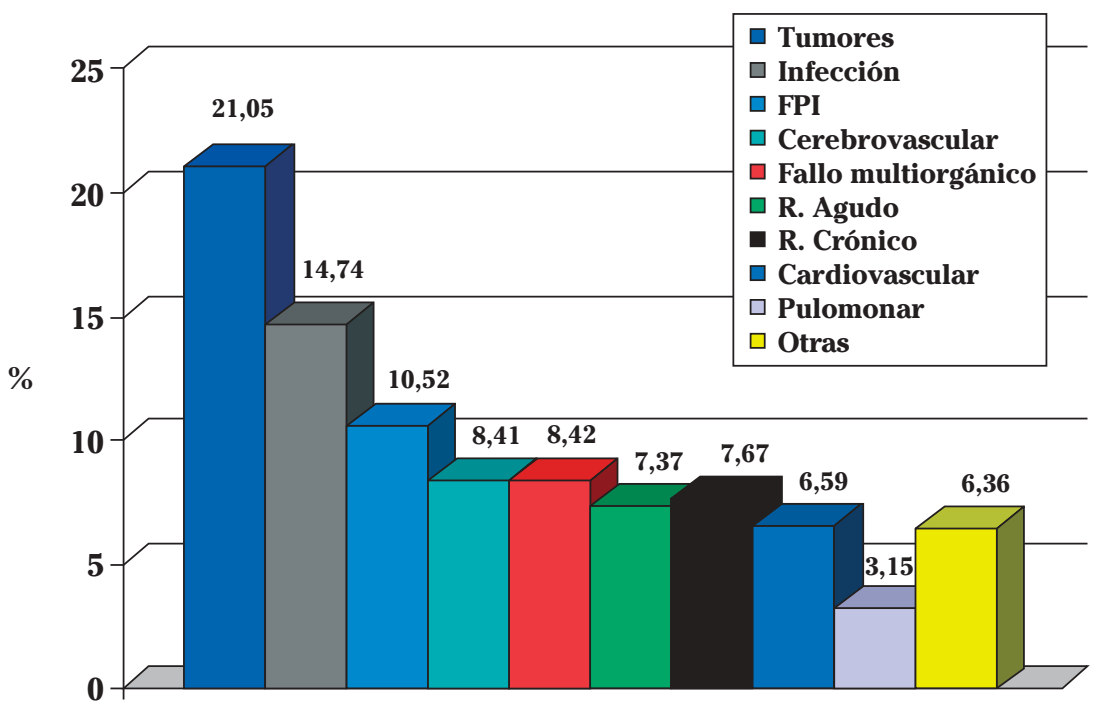

Figura 4. Causas total de muerte tras el trasplante. FPI (fallo primario del injerto).

ciencia cardiaca terminal, la oportunidad de recibir el único tratamiento eficaz para aumentar su supervivencia: el trasplante cardíaco.

\section{BIBLIOGRAFIA}

1. Aziz T, Burgess M, Khafagy R, Wynn Hann A, CAMPBEll C, RAHMAN A et al. Bicaval standard techniques in orthotopic heart transplantation: medium term experience in cardiac performance and survival. J Thorac Cardiovasc Surg 1999; 118: 115-122.

2. Sievers HH, Leyh R, Jahnke A, Petry A, KraAtz EG, Herrmann G et al. Bicaval versus atrial anastomoses in cardiac transplantation: right atrial dimension and tricuspid valve function at rest and during exercise up to thirty-six months after transplantation. J Thorac Cardiovasc Surg 1994; 108: 780-784.

3. El-Gamel A, Yonan NA, Grant S, DeiraniYa AK, RAHMAN AN, SARSAM MA et al. Orthotopic heart transplantation: a comparison between the standard and the bicaval Wythenshawe techniques. J Thorac Cardiovasc Surg 1995; 109: 721-730.

4. El-Gamel A, Deiraniya AK, Rahman AN, CampBELL CS, YONAN NA. Orthotopic heart transplantation hemodynamics: does atrial preservation improve cardiac output after transplantation? J Heart Lung Transpl 1996; 15: 564-571.

5. LeyH RG, JahnKe AW, KRAatz EG, Sievers HH. Cardiovascular dynamics and dimensions after bicaval and standard cardiac transplantation. Ann Thor Surg 1995; 59: 1495-1500.

6. Traversi E, Pozzoli M, Grande A, Forni G, AsSANDRI J, VigANo $M$ et al. The bicaval anastomosis technique for orthotopic heart transplantation yields better atrial function than the standard technique: an echocardiographic automatic boundary detection study. J Heart Lung Transplant 1998; 17: 1065-1074.

7. Wang SS, Chu SH, Hsu RB, Chen YS, Chou NK, Ko WJ. Is bicaval anastomosis superior to standard atrial procedure of heart transplantation? Transplant Proc 2000; 32: 23962397.

8. COPELAND JG. Heart transplantation: the standard versus bicaval technique controversy. Transplant Proc 2000; 32: 1519-1520.

9. Beniaminovitz A, Savoia MT, Oz M, GalantowICZ M, Di Tullio MR, Homma S et al. Improved atrial function in bicaval versus standard orthotopic techniques in cardiac transplantation. Am J Cardiol 1997; 80: 1631-1635.

10. Milano CA, Shah AS, Trigt PV et al. Transplantation of the heart. Adv Surg 1996; 2: 265. 
11. Milano CA, Shah AS, Van Trigt P, Davis RD, Glower DD, Higginbotham MB et al. Evaluation of early postoperative results after bicaval versus standard cardiac transplantation and review of the literature. Am Heart J 2000; 140: 717-721.

12. Brandt M, Harringer W, Hirt SW, Walluscheck KP, Cremer J, Sievers HH et al. Influence of bicaval anastomosis on late ocurrence of atrial arrythmia after heart transplantation. Ann Thorac Surg 1997; 64: 70-72.

13. Grande AM, Rinaldi M, D'Armini A, Campana C, Traversi E, Pederzoldi $\mathrm{C}$ et al. Orthotopic heart transplantation: standard versus bicaval technique. Am J Cardiol 2000; 85: 1329-1333.

14. Trento A, Takkenberg JM, Czer LSC, Blanche C, Nessim S, Cohen MH et al. Clinical experience with one hundred consecutive patients undergoing orthotopic heart transplantation with bicaval and pulmonary venous anastomosis. J Thorac Cardiovasc Surg 1996; 112: 1496-1503.

15. Blanche C, Nessim S, Quartel A, Takkenberg JJ, Aleksic I, Cohen M et al. Heart transplantation with bicaval and pulmonary venous anastomosis: a hemodynamic analysis of the first 117 patients. J Cardiovasc Surg 1997; 38 : 561-566.

16. Aleksic I, Czer LS, Freimark D, Takkenberg JJ, Dalichau H, Valenza M et al. Resting hemodynamics after total versus standard orthotopic heart transplantation. Thorac Cardiovasc Surg 1996; 44:193-198.

17. Bouchart F, Derumeaux G, Mouton-Schleifer D, Bessau JP, Redonnet M, Soyer R. Conventional and total orthotopic cardiac transplantation: a comparative clinical and echocardiographical study. Eur J Cardiothorac Surg 1997; 12: 555-559.

18. Bniaminovitz A, Itescu s, Lietz K, Donovan M, BurKe EM, GrofF BD et al. Prevention of rejection in cardiac transplantation by blockade of the interleukin-2 receptor with a monoclonal antibody. N Engl J Med 2000; 342: 647-648.

19. Hershberger RE, Starling RC, Eisen HJ, Bergh $\mathrm{CH}$, Kormos RL, Love RB et al. Daclizumab to prevent rejection after cardiac transplantation. N Engl J Med 2005; 352: 2749-2750.

20. TAylor DO, Bristow MR, O'Connell JB, Price GD, Hammond EH, Doty DB et al. Improved long-term survival after heart transplantation predicted by successful early withdrawal from maintenance corticosteroid therapy. J Heart Lung Transplant 1996; 15: 1039-1046.

21. TAYlor DO, EdWards LB, BouceK MM, Trulock EP, Deng MC, KECK BM et al. Registry of the
International Society for Heart and Lung Transplantation: twenty-second official adult heart transplant report-2005. J Heart Lung Transplant 2005; 24: 945-955.

22. Grossi P, Farina C, Fiocchi R, Dalla Gasperina D. Prevalence and outcome of invasive fungal infections in 1963 thoracic organ transplant recipients: a multicenter retrospective study. Italian Study Group of Fungal Infections in Thoracic Organ Transplant Recipients. Transplantation 2000; 70: 112-116.

23. AlexAnder BD. Prophylaxis of invasive mycoses in solid organ transplantation. Curr Opin Infect Dis 2002; 15: 583-589.

24. Playford EG, Webster AC, Sorrell tC, Craig JC. Antifungal agents for preventing fungal infections in non-neutropenic critically ill and surgical patients: systematic review and meta-analysis of randomized clinicaltrials. J Antimicrob Chemother 2006; 57: 628-638.

25. Kubo, SH, Naftel, DC, Mills, RM JR, O`DonNELL J, RoDehefFER RJ, CinTRON GB et al. Risk factors for late recurrent rejection after heart transplantation: a multiinstitutional, multivariable analysis. Cardiac Transplant Research Database Group. J Heart Lung Transplant 1995; 14: 409.

26. KobashigaWa JA, Kirkilin JK, Naftel DC, Bourge RC, Ventura HO, Mohanty PK et al. Pretransplantion risk factors for acute rejection after cardiac transplantation: A multiinstitutional study.The Transplant Cardiologist Research Database Group. J Heart Lung Transplant 1993; 12: 355-366.

27. Jarcho J, Naftel DC, Shroyer TW, Kirklin JK, BOURGE RC, BARR ML et al. Influence of HLA mismatch on rejection after heart transplantation: a multiinstitutional study. The Cardiac Transplant Research Database Group. J Heart Lung Transplant 1994; 13: 583-595; discussion 595-596.

28. Mills RM, Naftel DC, KiRKLin JK, et al. Heart transplant rejection with hemodynamic compromise: a multiinstitutional study of the role of endomyocardial cellular infiltrate. Cardiac Transplant Research Database. J Heart Lung Transplant 1997; 16: 813-821.

29. Cui G, Tung T, Kobashigawa J, Laks H, Sen L. Increased incidence of atrial flutter associated with the rejection of heart transplantation. Am J Cardiol 2001; 88: 280-284.

30. SteWART S, WinTER GL, Fisbein MC. Revision on the 1990 working formulation for the standardization of nomenclature in the diagnosis of Heart rejection. ISHLT Consensus report J Heart Lung Transplant 2005; 24: 1710-1720. 
31. Behr TM, Feucht HE, Richter K, Reiter C, Spes $\mathrm{CH}$, Pongratz D et al. Detection of humoral rejection in human cardiac allografts by assessing the capillary deposition of complement fragment C4d in endomyocardial biopsies. J Heart Lung Transplant 1999; 18: 904-912.

32. Michaels PJ, Espejo ML, Kobashigawa J, Alejos JC, Burch C, TAKEMOTO S et al. Humoral rejection in cardiac transplantation: risk factors, hemodynamic consequences and relationship to transplant coronary artery disease. J Heart Lung Transplant 2003; 22: 58-69.

33. Fishbein M, KobashigaWa J. Biopsy-negative cardiac transplant rejection: etiology, diagnosis and therapy. Curr Opin Cardiol 2004; 19: 166-169.

34. Klingenberg R, Koch A, Schnabel PA, ZimmerMANN R, SACK FU, HAASS M et al. Rejection of ISHLT grade $\geq 3 \mathrm{~A}$ occurring late after heart transplantation-a distinct entity? J heart Lung Transpl 2003; 22: 1005-1013.

35. Fishbein M, Kobashigawa J. Biopsy-negative cardiac transplant rejection: etiology, diagnosis and therapy. Curr Opin Cardiol 2004; 19: 166-169.

36. Rose ML, Smith J, Dureau G, Keogh A, KoBASHigowa J. Mycophenolate mofetil decreases antibody production after cardiac transplantation. J Heart Lung Transpl 2002; 21: 282-285.

37. TAYlor DO, EdWARds LB, BouceK MM, Trulock EP, Deng MC, Keck BM et al. Registry of the International Society for Heart and Lung Transplantation: twenty-second official adult heart transplant report-2005. J Heart Lung Transplant 2005; 24: 945-955.

38. Costanzo MR, Naftel DC, Pritzker MR, HeILMAN JK 3RD, BOEHMER JP, BROZENA SC et al. Heart transplant coronary artery disease detected by coronary angiography: a multiinstitutional study of preoperative donor and recipient risk factors. Cardiac Transplant Research Database. J Heart Lung Transplant 1998; 17: 744-753.

39. Tuzcu EM, Hobbs RE, Rincon G, Bott-Silverman C, DE Franco AC, Robinson $\mathrm{K}$ et al. Occult and frequent transmission of atherosclerotic coronary disease with cardiac transplantation. Insights from intravascular ultrasound. Circulation 1995; 91: 17061713.

40. Tuzcu EM, Kapadia SR, Sachar R, Ziada KM, CROWE TD, FENG J et al. Intravascular ultrasound evidence of angiographically silent progression in coronary atherosclerosis predicts long-term morbidity and mortality after cardiac transplantation. J Am Coll Cardiol 2005; 45: 1538-1542.
41. Kobashigawa JA, Tobis JM, Starling RC, Tuzcu EM, SMith AL, VAlantine HA et al. Multicenter intravascular ultrasound validation study among heart transplant recipients: outcomes after five years. J Am Coll Cardiol 2005; 45: 1532-1537.

42. Tuzcu EM, De Franco AC, Goormastic M, Hobbs RE, Rincon G, BotT-SiLverman C et al. Dichotomous pattern of coronary atherosclerosis 1 to 9 years after transplantation: insights from systematic intravascular ultrasound imaging. J Am Coll Cardiol 1996; 27: 839-846.

43. Valantine H. Cardiac allograft vasculopathy after heart transplantation: risk factors and management J Heart Lung Transplant 2004; 23(5S): S187-193.

44. Wenke K, Meiser B, Thiery J, Nagel D, von Scheidt W, SteinBeck G et al. Simvastatin reduces graft vessel disease and mortality after heart transplantation: a four-year randomized trial. Circulation 1997; 96: 13981402.

45. Kobashigawa JA, Katznelson S, LaKs H, Johnson JA, YeATMAN L, WANG XM et al. Effect of pravastatin on outcomes after cardiac transplantation. N Engl J Med 1995; 333: 621-627.

46. Schroeder JS, Gao SZ, Alderman EL, Hunt SA, Johnstone I, Boothroyd DB et al. A preliminary study of diltiazem in the prevention of coronary artery disease in heart-transplant recipients. N Engl J Med 1993; 328: 164-170.

47. Schroeder JS, GAO SZ. Calcium blockers and atherosclerosis: lessons from the Stanford Transplant Coronary Artery Disease/Diltiazem Trial. Can J Cardiol 1995; 11: 710-715.

48. FAng JC, Kinlay S, Beltrame J, Hikiti H, WainSTEIN M, BEHRENDT D et al. Effect of vitamins C and $\mathrm{E}$ on progression of transplant-associated arteriosclerosis: a randomised trial. Lancet 2002; 359: 1108-1113.

49. Eisen HJ, Tuzcu EM, Dorent R, Kobashigawa J, Mancini D, Valantine-Von Kaeppler HA et al. Everolimus for the prevention of allograft rejection and vasculopathy in cardiac-transplant recipients. N Engl J Med 2003; 349: 847858.

50. Keogh A, Richardson M, Ruygrok P, Spratt P, Galbraith A, O'Driscoll G et al. Sirolimus in de novo heart transplant recipients reduces acute rejection and prevents coronary artery disease at 2 years: a randomized clinical trial. Circulation 2004; 110: 2694-2700.

51. Lamich R, Ballester M, Marti V, Brossa V, AYMAT R, CARRIO I et al. Efficacy of augmented immunosuppressive therapy for early vas- 
culopathy in heart transplantation. J Am Coll Cardiol 1998; 32: 413-419.

52. Mancini D, Pinney S, BuRkhoff D, LaManca J, ITESCU S, BURKE E et al. Use of rapamycin slows progression of cardiac transplantation vasculopathy. Circulation 2003; 108: 48-53.

53. BenZa RL, Zoghbi GJ, TAllaj J, Brown R, KIRKLIN JK, HUBBARD M. Palliation of allograft vasculopathy with transluminal angioplasty: a decade of experience. J Am Coll Cardiol 2004; 43: 1973-1981.

54. Halle AA $3^{\text {po }}$, DiSciascio G, Massin EK, Wilson RF, Johnson MR, Sullivan HJ et al. Coronary angioplasty, atherectomy and bypass surgery in cardiac transplant recipients. J Am Coll Cardiol 1995; 26: 120-128.

55. Scholl FG, Sen L, Drinkwater DC, LaKs H, Ma XY, HoNG YS et al. Effects of human tissue plasminogen gene transfer on allograft coronary atherosclerosis. J Heart Lung Transplant 2001; 20: 322-329.

56. Iwata A, Sai S, Moore M, Nyhuis J, DE FriesHallstrand R, QuetingCo GC et al. Gene therapy of transplant arteriopathy by liposome- mediated transfection of endothelial nitric oxide synthase. J Heart Lung Transplant 2000; 19: 1017-1028.

57. VIAL T, Descotes J. Immunosuppressive drugs and cancer. Toxicology 2003; 185: 229-240.

58. KEOGH A. Long-term benefits of mycophenolate mofetil after heart transplantation. Transplantation 2005; 79: S45-46.

59. Guba M, von Breitenbuch P, Steinbauer M, Koenl G, Flegel S, Hornung $M$ et al. Rapamycin inhibits primary and metastatic tumor growth by antiangiogenesis: involvement of vascular endothelial growth factor. Nat Med 2002; 8: 128-135.

60. Stallone G, Schena A, Infante B, Di Paolo S, Loverre A, Maggio G et al. Sirolimus for Kaposi's sarcoma in renal-transplant recipients. N Engl J Med 2005; 352: 1317-1323.

61. Koenl GE, Andrassy J, Guba M, Richter S, KROEMER A, SCHERER MN et al. Rapamycin protects allografts from rejection while simultaneously attacking tumors in immunosuppressed mice. Transplantation 2004; 77 : 1319-1326. 